\title{
Peak Effect and Dynamic Melting of Vortex Matter in $\mathrm{NbSe}_{2}$ Crystals
}

\author{
N. Kokubo, K. Kadowaki, and K. Takita \\ Institute of Materials Science, University of Tsukuba, 1-1-1, Tennoudai, Tsukuba, Ibaraki 305-8573, Japan
}

(Received 1 July 2005; published 19 October 2005)

\begin{abstract}
We present a mode locking (ML) phenomenon of vortex matter observed around the peak effect regime of $2 \mathrm{H}-\mathrm{NbSe}_{2}$ pure single crystals. The ML features allow us not only to trace how the shear rigidity of driven vortices persists on approaching the second critical field, but also to demonstrate a dynamic melting transition of driven vortices at a given velocity. We observe the velocity dependent melting signatures in the peak effect regime, which reveal a crossover between the disorder-induced transition at small velocity and the thermally induced transition at large velocity. This uncovers the relationship between the peak effect and the thermal melting.
\end{abstract}

Discontinuous jumps in equilibrium magnetization observed well below the mean field line in clean high $T_{c}$ cuprate superconductors [1] have been widely recognized as a hallmark of the thermodynamic melting transition (MT), which separates a vortex solid state, where elastic interaction dominates and quasi-long-ranged crystalline correlations develop in vortex structure from a vortex liquid state in which thermal fluctuations disrupt the crystalline order and the shear rigidity vanishes.

The melting signature is often accompanied by the pronounced peak anomaly of the magnetization or critical current [2], known as the peak effect (PE), originating from the rapid softening of the vortex lattice and the random pinning potential due to disorder quenched in a host material. The close proximity of the MT on the PE has led to a reexamination of the physical properties close to the second critical field $H_{c 2}$, especially on low $T_{c}$ materials like $\mathrm{Nb}$ [3-5] and $\mathrm{NbSe}_{2}$ [6,7]. Various experimental results evidence the pinning-induced structural transformation into a disordered array of vortices, indicating the dominant influence of the quenched disorder in the PE regime [3,7-9]. However, the presence of the MT and its relation with the PE remain controversial [4,5].

The effect of the quenched disorder changes remarkably when vortices are driven by a transport current. As proposed theoretically [10-12] and experimentally $[6,13,14]$, at low drive the "shaking action" of the random pinning due to the quenched disorder disrupts largely the internal periodicity in the moving structure of driven vortices, while at high drive the influence of the disorder diminishes and the elastic interaction becomes dominant, resulting in moving solid states at large velocity.

This leads to an unique opportunity to study whether the "driven lattice" undergoes thermally induced MT and also whether the transition occurs in the disorder dominant PE regime. In this Letter, we present experimental evidences for the dynamic melting transition (DMT) of driven lattice observed just above the PE of $\mathrm{NbSe}_{2}$ by means of a mode locking (ML) experiment. ML is a dynamic synchronization between rf drive superimposed on the dc drive and collective lattice (elastic) modes excited over driven vortices at internal frequencies $f_{\text {int }}=q v / a$ with the average velocity $v$, the lattice spacing $a$ and an integer $q[15,16]$. When the internal frequency and the external drive frequency are harmonically related, i.e., $f_{\text {int }}=p f$ or $v=$ $(p / q) f a$ with an integer $p$, the elastic modes are dynamically locked on rf drive. We observe this dynamic resonance as multiple current steps in a current-voltage $(I V)$ curve or multiple peaks in a differential conductance $(d I / d V)$ curve. The ML features allow us not only to trace how the shear rigidity of driven vortices persists on approaching $H_{c 2}$, but also to demonstrate the DMT of driven vortices at a given velocity. We will show that the velocity dependence of dynamic melting signatures unveils the relationship between the PE and the MT.

Platelets of $2 \mathrm{H}-\mathrm{NbSe}_{2}$ pure single crystal used in this study were grown by an iodine vapor transport method [17]. The thinnest three crystals (typically $10 \mu \mathrm{m}$ thick) were cut in the bar shape and cleaved with no significant optical surface damage. Contacts for the fourprobe method were made by indium solder. The crystals have the superconducting transition temperature $T_{c}$ of $7.2 \mathrm{~K}$ determined from the midpoint of resistive transition. The transition width is about $50 \mathrm{mK}$ between $10 \%$ and $90 \%$ of the normal state resistance just above $T_{c}$ [e.g., $R(7.4 \mathrm{~K})]$. Typical values of residual resistance ratio $R(295 \mathrm{~K}) / R(7.4 \mathrm{~K})$ for the crystals are ranged between 30 and 40 . However, no significant change of the room temperature resistivity $\rho_{0}$ of $1.2 \times 10^{-6} \Omega \mathrm{m}$ was observed in crystals from different batches [17]. This allows us to estimate the thickness of the cleaved crystals from the room temperature resistance $R(295 \mathrm{~K})$.

The measurements discussed in this study were carried out on a cleaved crystal with a dimension of $0.72 \mathrm{~mm}(l) \times$ $0.63 \mathrm{~mm}(w) \times 0.9(t) \mu \mathrm{m}$. Figure 1(b) shows results of $H_{c 2}$ on the crystal obtained from a measurement of fluxflow resistance with magnetic field applied perpendicularly to the platelet. As displayed in the inset to Fig. 1(a), $H_{c 2}$ is determined from the intersectional point between the linear extrapolation of flow resistance indicated by a solid line 


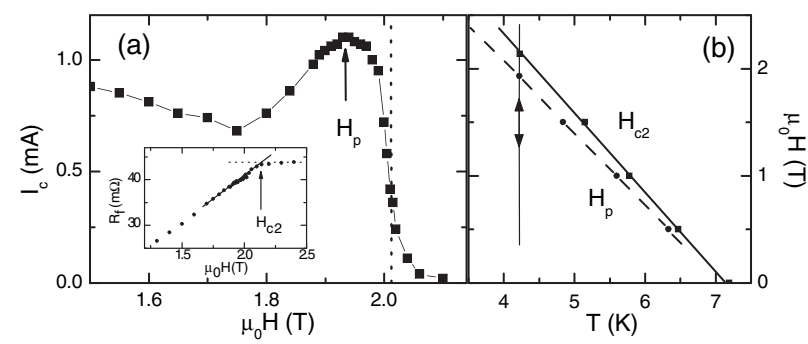

FIG. 1. (a) Peak anomaly of critical current $I_{c}$ measured by varying magnetic field $H$ at $4.2 \mathrm{~K}$. The peak field $H_{p}$ is denoted by an arrow. A dotted line marks the dynamic melting field measured at high velocity. The inset displays how differential resistance $R_{d}$ measured at a large dc current of $30 \mathrm{~mA}\left(\gg I_{c}\right)$ depends on field. The definition of the second critical field $H_{c 2}$ is given in text. (b) Phase diagram of $\mathrm{NbSe}_{2}$. Both $H_{c 2}$ and $H_{p}$ are plotted. See text for arrows.

and the normal resistance by a dotted line [18]. The slope of $H_{c 2}$ on $T$ at $T_{c}(\equiv-S)$ is $-0.72 \mathrm{~T} / \mathrm{K}$, in good agreement with magnetization results $[7,17,19]$. Shown in Fig. 1(a) is a plot of the critical current $I_{c}$ vs magnetic field $H$, obtained by ramping the field up (and also down) after cooling the crystal to $4.2 \mathrm{~K}$ in the zero field [indicated by arrows in Fig. 1(b)] [20]. Here, $I_{c}$ is determined from $I V$ curves by a $10 \mu \mathrm{V} / \mathrm{m}$ criterion. It exhibits a peak behavior below $\mu_{0} H_{c 2}$ of $2.14 \mathrm{~T}$, characterized by the peak field $H_{p}$ of $1.93 \mathrm{~T}$. We note that, because of inhomogeneous edges in the strip geometry, $I_{c}$ below $H_{p}$ has both bulk and edge contributions [21,22], resulting in broadening for the lower part of the PE.

Figure 2(a) shows a series of $d I / d V-V$ curves measured in $1.90 \mathrm{~T}$ (just below $H_{p}$ ) with superimposing $3 \mathrm{MHz}$ rf current of various amplitudes [23]. Application of a relatively large rf current of $16.7 \mathrm{~mA}$, for instance, induces clear conductance ML peaks at equidistant voltages. The first peak corresponds to the fundamental $(p / q=1 / 1)$ and others are higher harmonics $(2 / 1$, and 3/1). Small peaks at subharmonic conditions with $q>1$ are also detected. As expected in the velocity condition $[v=(p / q) f a]$, those resonance voltages $(\propto v)$ increase linearly with rf drive frequency [see Fig. 2(b)]. Thus, by changing $f$, we can detect the shear rigidity of driven vortices at various velocities.

In Fig. 2(c), we show how the fundamental resonance depends on rf current $I_{\mathrm{rf}}$. Here, the fundamental ML current width $\Delta I$ is obtained by integrating the conductance ML peak with respect to the flux-flow baseline [see the inset to Fig. 2(c)]. It shows an oscillatory behavior with $I_{\mathrm{rf}}$, which agrees qualitatively with the squared Bessel function of the first kind displayed by a broken curve. This behavior is expected when the random pinning due to the quenched disorder excites elastic modes in driven elastic lattices [15]. In other frequencies and fields, similar oscillatory behavior of $\Delta I$ on $I_{\mathrm{rf}}$ is observed in different $\mathrm{rf}$ current scales. For simplicity, we focus mainly on the first
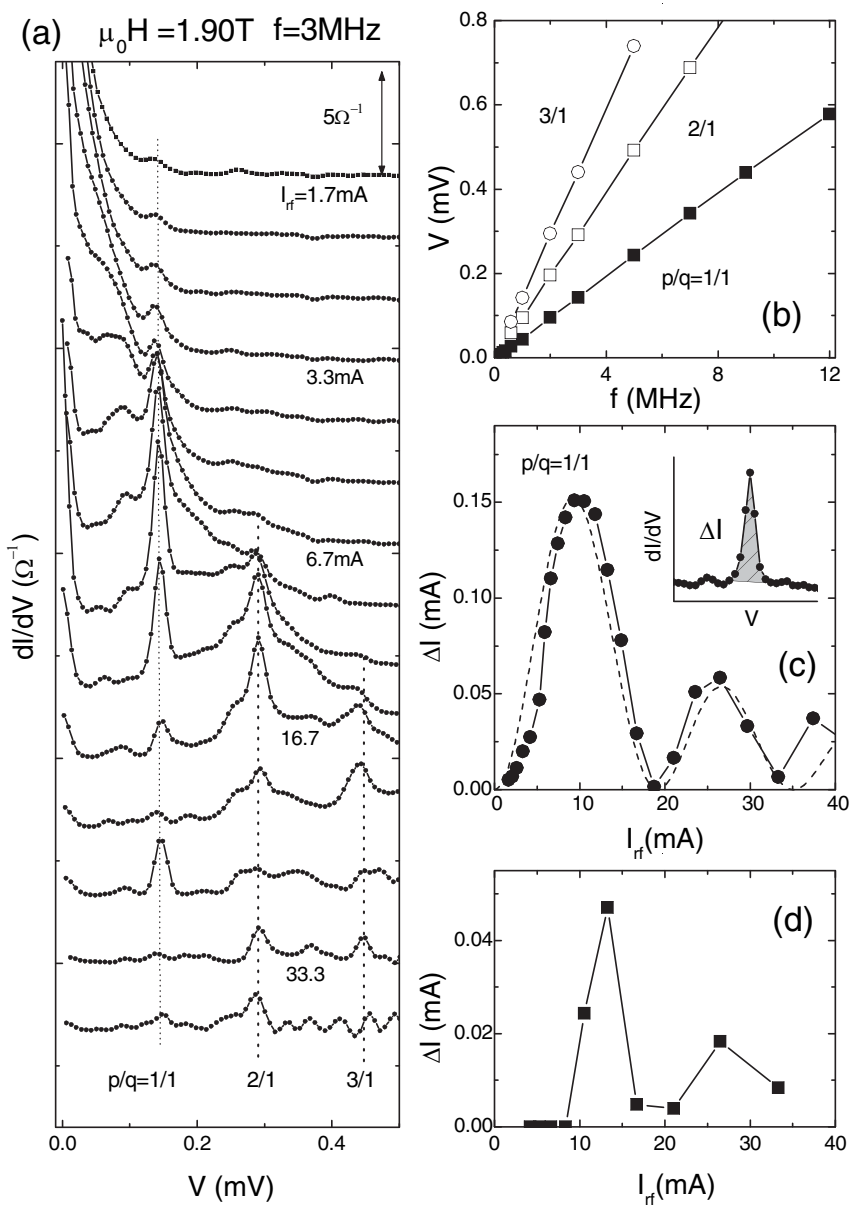

FIG. 2. ML phenomenon of driven vortices. (a) Differential conductance $(d I / d V)$-voltage $(V)$ curves measured in $1.90 \mathrm{~T}$ with superimposing $3 \mathrm{MHz}$ rf current of various amplitudes. For clarity, the origin for each curve is shifted vertically. ML conditions are denoted. (b) Frequency, $f$, dependence of ML voltages for the fundamental and higher harmonics. (c) rf current $I_{\mathrm{rf}}$ vs the fundamental ML current width $\Delta I$ obtained by integrating the fundamental ML peak shown in (a) with respect to the fluxflow baseline (see the inset). See text for a broken curve. (d) $\Delta I$ for $3 \mathrm{MHz}$ vs $I_{\mathrm{rf}}$ observed in $1.995 \mathrm{~T}$ between $H^{*}$ and $H_{\mathrm{on}}$.

maximum $\Delta I_{\max }$ of the fundamental ML width in the following analysis.

Next, we turn to the magnetic field dependence of the ML resonance. In Fig. 3(a), we show how the fundamental ML voltage $V_{1 / 1}$ evolves with $H$. As observed, $V_{1 / 1}$ for $7 \mathrm{MHz}$ increases with $H$ with downward curvature. This is in good quantitative agreement with the fundamental ML-voltage condition displayed by a solid curve; $V_{1 / 1}=A f \Phi_{0} l / a_{0}$ with the equilibrium lattice spacing $a_{0}\left(=\sqrt{A \Phi_{0} / B}\right)$, a factor $A(=2 / \sqrt{3})$, the sample length $l(=0.72 \mathrm{~mm})$, and the vortex density $B=\mu_{0} H$. Note that no fitting parameter is used for this comparison.

As the magnetic field approaches $\mu_{0} H_{c 2}(=2.14 \mathrm{~T})$, however, the ML resonance suddenly disappears at a certain field. An example is displayed in Fig. 3(b), where the 

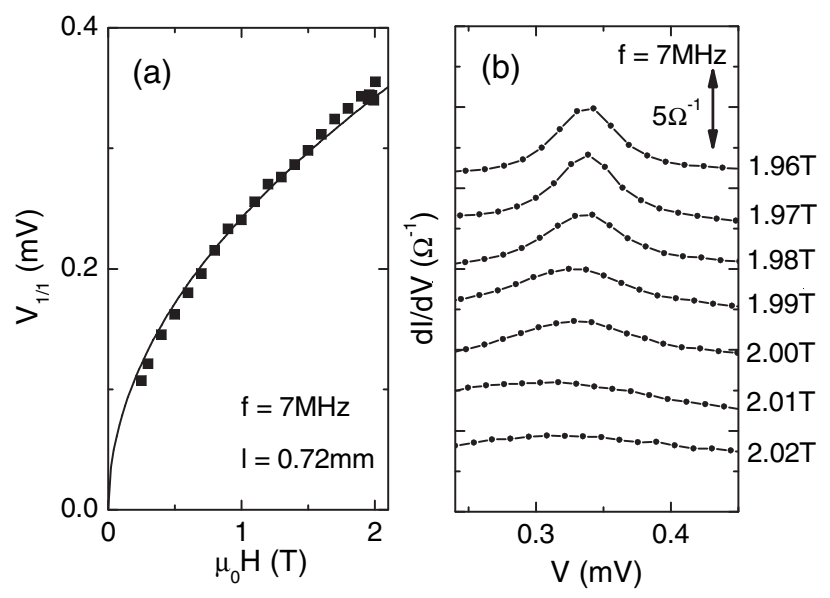

FIG. 3. (a) Magnetic field dependence of the fundamental ML voltage $V_{1 / 1}$ measured with superimposing a $7 \mathrm{MHz}$ rf current. A solid curve represents the fundamental ML-voltage condition (see text). (b) Differential conductance curves measured with a $7 \mathrm{MHz}$ rf current in fields from $1.96 \mathrm{~T}$ to $2.02 \mathrm{~T}$ by a constant field step of $0.01 \mathrm{~T}$. For clarity the origin for each curve is vertically shifted.

$d I / d V$ curves measured with superimposing a $7 \mathrm{MHz}$ rf current in different fields are shown. The fundamental ML peak is clearly observed in lower fields. As $H$ is ramped up, the peak becomes small and it seems to vanish around $\mu_{0} H=2.01 \mathrm{~T}$, above which the $d I / d V$ curves become featureless.

The characteristics are better displayed by plotting $\Delta I_{\max }$ against the field, which is given in Fig. 4(a). $\Delta I_{\max }$ for $3 \mathrm{MHz}$, for instance, starts to show a rapid drop around $1.98 \mathrm{~T}$ (defined as the onset field $H_{\mathrm{on}}$ ) and disappears around $2.00 \mathrm{~T}$, while it is insensitive to $H$ below $H_{\text {on }}$. We define the vanishing field $H^{*}$ from the linear extrapolation

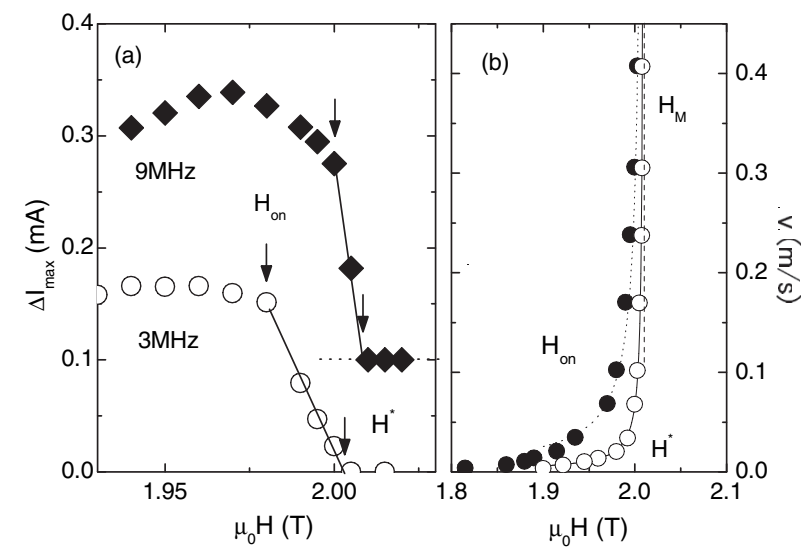

FIG. 4. (a) Magnetic field vs the maximized fundamental ML current width $\Delta I_{\max }$ for 3 and $9 \mathrm{MHz}$. For clarity, the origin for $9 \mathrm{MHz}$ data is shifted vertically. Onset $H_{\mathrm{on}}$ and vanishing $H^{*}$ fields are indicated by arrows. Their velocity dependencies are given in (b). A broken line represents the saturation field. See text for solid and dotted curves. to $\Delta I_{\max }=0$ as indicated by a solid line. Above this field, no ML resonance appears at any amplitude of $I_{\mathrm{rf}}$, indicating the absence of the shear rigidity in driven vortices. Thus, the vanishing field should mark the DMT from coherent to liquidlike incoherent flow states $[15,16]$. We note that this phenomenon emerges just above the PE where $I_{c}$ drops, and therefore it should be driven by the thermal fluctuations, in addition to the quenched disorder.

The reduction of the disorder effect is expected in the ML features measured at high frequencies (large velocities). As shown in Fig. 4(a), $\Delta I_{\max }$ measured at a high frequency of $9 \mathrm{MHz}$ exhibits a sharper drop at slightly higher fields. Namely, both the onset and the vanishing fields increase slightly, while the field range where the linear drop of the ML width occurs becomes narrow. We observe even sharper phenomenon at high frequencies, indicating the sudden disappearance of the shear rigidity in driven vortices at large velocities.

The striking frequency (velocity) dependence is also observed in the onset and vanishing fields, characterizing the dynamic melting signatures. In Fig. 4(b) results of $H^{*}$ and $H_{\mathrm{on}}$ are plotted against velocity determined from the fundamental velocity condition of $v=f a_{0}$. As observed, $H^{*}$ (open symbols) increases with $v$ and exhibits a saturation behavior toward $\approx 2.01 \mathrm{~T}$ at larger velocities. $H_{\mathrm{on}}$ (solid symbols) also exhibits a similar saturation behavior toward the nearly identical field at larger velocities. The difference between $H^{*}$ and $H_{\text {on }}$ at a constant velocity decreases with increasing $v$ and becomes negligible experimentally at large velocity. These behaviors imply that at small velocity the influence of the disorder facilitates the occurrence of the DMT, while at large velocity it becomes negligible and the thermal effect dominates on the phenomenon. Thus, the velocity dependence of the dynamic melting signatures reveals a crossover between the disorder-driven and the thermally driven transitions.

Regarding the vanishing field at a given velocity as a crystallization velocity $v_{c}$ at the field, the saturation behavior is qualitatively similar to the dynamic ordering picture proposed by Koshelev and Vinokur [10], in which combined influences of the thermal fluctuations and the quenched disorder on vortex dynamics near the thermodynamic melting transition are taken into account. In this model, the crystallization velocity diverges as $v_{c}=$ $v_{0} /\left(1-T / T_{M}\right)$ on approaching the melting point $T_{M}$ from below. As displayed by a solid curve, the $H^{*}$ data are well approximated by a similar function of $v_{c}=$ $v_{0} /\left(1-H / H_{M}\right)^{\alpha}$ with the melting field $\mu_{0} H_{M}=2.01 \mathrm{~T}$ (indicated by a broken line), $\alpha=1$ and $v_{0}=0.33 \mathrm{~mm} / \mathrm{s}$ over nearly two decades of velocity [24]. This allow us to identify the saturation field as the thermodynamic melting point for the vortex lattice. Thus, the sharp drop of ML by the saturation field signifies the thermally induced DMT of driven lattice.

Let us compare the saturation field to a recent quantitative theoretical result for the thermodynamic melting line 
proposed by Li and Rosenstein [25]. The MT is set in when the lowest Landau-level-scaled temperature $a_{T}=$ $-\left(\sqrt{N_{G i} / 2} \pi t h\right)^{-2 / 3}(1-t-h)=-9.5$, with their Ginzburg number $N_{G i}=\left(\left[k_{B} T_{c} \gamma / 4 \pi^{2} \mu_{0} H_{c}(0)^{2} \xi^{3}\right]^{2}\right) / 2$, a reduced temperature $t=T / T_{c}$, and a reduced field $h=$ $H / H_{c 2}(0)$. Using the anisotropy parameter $\gamma=3$ [19], the Ginzburg-Landau (GL) thermodynamic field at $T=$ $0 \mu_{0} H_{c}(0)=0.24 \mathrm{~T}$ [19], and the GL second critical field $\mu_{0} H_{c 2}(0)=S T_{c}=5.18 \mathrm{~T}$ for the present crystal, we find that the saturation field of $2.01 \mathrm{~T}$ at $4.2 \mathrm{~K}$ gives $a_{T}=-10$. A nearly similar result of $a_{T}=-9$ is also found in other crystal with $\mu_{0} H_{M}=2.02 \mathrm{~T}$ at $4.2 \mathrm{~K}$. These are in good agreement with the theoretical result.

Next, we discuss flow states near the DMT. In the regime between the onset and vanishing fields, an anomalous dependence of $\Delta I$ on $I_{\mathrm{rf}}$ is observed, of which an example is shown in Fig. 2(d). There is a minimum rf current to observe ML, indicating the influence of the quenched disorder disrupting the shear rigidity in driven vortices. Such threshold behavior in ML favors with a smectic flow (or a layered liquid) state $[26,27]$, in which only the periodicity perpendicular to the flow direction is preserved. Thus, the $H^{*}$ curve would mark a dynamic transition between the smectic and the liquid flow states. Meanwhile, for $H<H_{\text {on }}$, the threshold rf current for ML disappears and $\Delta I$ shows the Bessel function like oscillatory behavior on $I_{\mathrm{rf}}$, suggesting a moving solid state. Thus, the solid-smectic transition would occur at the $H_{\text {on }}$ curve. A similar two step process of the DMT has been proposed theoretically $[11,12]$.

Finally, we compare the melting curve of $H^{*}$ to the peak behavior of $I_{c}$. As indicated by a dotted line in Fig. 1(a), the dynamic melting point (or $H_{M}$ ) at large velocity emerges in the higher part of the PE. On reducing velocity, $H^{*}$ approaches $H_{p}$ and seems to disappear (or be below our experimental resolutions) around $1.9 \mathrm{~T}$ just below $H_{p}$. A recent study on edge and bulk transport [22] reveals that the bulk component of $I_{c}$ begins to show a peak anomaly just below the peak temperature of $I_{c}$. This prompts us to relate the disappearance of $H^{*}$ to the disordering transition for the PE. Thus, the $H^{*}$ curve would be a locus of the melting point connecting smoothly the PE with the MT as a function of the disorder effect (velocity).

In summary, employing ML techniques, we have presented experimental evidences for the DMT of a driven vortex lattice observed just above the $\mathrm{PE}$ of $2 \mathrm{H}-\mathrm{NbSe}_{2}$ single crystals. At small velocity the transition driven predominantly by disorder occurs near $H_{p}$, while at large velocity the transition by thermal fluctuations is observed by $H_{M}\left(>H_{p}\right)$. The velocity dependence of the melting signatures reveals the smooth crossover between the PE and the MT.
N.K. used facilities in the cryogenic center in the University of Tsukuba. N. K. thanks P. H. Kes, A. E. Koshelev, and T. Nishizaki for useful comments. This work was partly supported by the Grant in Aid for Scientific Research (Grant No. 16710063) from the Ministry of Education, Science and Culture, Japan.

[1] E. Zeldov et al., Nature (London) 375, 373 (1995).

[2] J. Shi et al., Phys. Rev. B 60, R12 593 (1999).

[3] P. L. Gammel et al., Phys. Rev. Lett. 80, 833 (1998).

[4] X. S. Ling et al., Phys. Rev. Lett. 86, 712 (2001).

[5] E. M. Forgan et al., Phys. Rev. Lett. 88, 167003 (2002).

[6] S. Bhattacharya and M. J. Higgins, Phys. Rev. Lett. 70, 2617 (1993); Physica C (Amsterdam) 257, 232 (1996).

[7] A. M. Troyanovski et al., Phys. Rev. Lett. 89, 147006 (2002).

[8] Y. Paltiel et al., Phys. Rev. Lett. 85, 3712 (2000).

[9] M. Marchevsky, M. J. Higgins, and S. Bhattacharya, Nature (London) 409, 591 (2001).

[10] A. E. Koshelev and V. M. Vinokur, Phys. Rev. Lett. 73, 3580 (1994).

[11] P. Le Doussal and T. Giamarchi, Phys. Rev. B 57, 11356 (1998).

[12] S. Scheidl and V.M. Vinokur, Phys. Rev. E 57, 2574 (1998); Phys. Rev. B 57, 13800 (1998).

[13] U. Yaron et al., Nature (London) 376, 753 (1995).

[14] M. C. Hellerqvist et al., Phys. Rev. Lett. 76, 4022 (1996).

[15] N. Kokubo et al., Phys. Rev. Lett. 88, 247004 (2002); N. Kokubo, R. Besseling, and P. H. Kes, Phys. Rev. B 69, 064504 (2004), and references there in.

[16] R. Besseling, N. Kokubo, and P. H. Kes, Phys. Rev. Lett. 91, 177002 (2003).

[17] K. Takita and K. Masuda, J. Low Temp. Phys. 58, 127 (1985).

[18] P. Berghuis and P. H. Kes, Phys. Rev. B 47, 262 (1993).

[19] N. Kobayashi, K. Noto, and Y. Muto, J. Low Temp. Phys. 27, 217 (1977), and references therein.

[20] During ML experiments, the crystals were immersed in liquid ${ }^{4} \mathrm{He}$ to avoid heating.

[21] Y. Paltiel et al., Phys. Rev. B 58, R14 763 (1998).

[22] Z. L. Xiao, Phys. Rev. B 65, 094511 (2002).

[23] Flux-flow skin depth defined as $\sqrt{2 \rho_{f} / 2 \pi f \mu_{0}}$ with fluxflow resistivity $\rho_{f}$ is $15 \mu \mathrm{m}$ at $3 \mathrm{MHz}$ and $1.9 \mathrm{~T}$.

[24] We also see similar agreement of the $H_{\text {on }}$ data $(>1.9 \mathrm{~T})$ with the identical function by $\mu_{0} H_{M}=2.01 \mathrm{~T}$ and a large value of by $v_{0}=1.4 \mathrm{~mm} / \mathrm{s}$ displayed by a broken curve.

[25] D. Li and B. Rosenstein, Phys. Rev. B 65, 220504(R) (2002).

[26] L. Balents, M. C. Marchetti, and L. Radzihovsky, Phys. Rev. B 57, 7705 (1998).

[27] A. B. Kolton, D. Dominguez, and N. Gronbech-Jensen, Phys. Rev. Lett. 86, 4112 (2001). 